\title{
Nutrient Intakes According to Pain in Patients with Arthritis
}

\author{
Yeong-Mi Seo ${ }^{1}$, Tae-Kyung $\mathrm{Kim}^{2}$, and Won-Hee $\mathrm{Choi}^{3}$ \\ ${ }^{I}$ Dept. of Nursing, Gyeongnam National University of Science and Technology, Jinju, \\ South Korea \\ ${ }^{2}$ Dept. of Nursing, Dong-Eui Institute of Technology College, Busan, South Korea \\ ${ }^{3}$ Dept. of Nursing, Kyungsung University, Busan, South Korea \\ Iasfirst@chol.com, ${ }^{2}$ phoebetk@dit.ac.kr, ${ }^{3}$ whchoi@ks.ac.kr
}

\begin{abstract}
Arthritis is the third most common chronic disease in Korea and causes severe pain and discomfort. This study utilized the data from the 5th National Health and Nutrition Survey. It is known that inappropriate nutritional status among the causes of pain in arthritis patients is caused by energy supply disturbance. In this study, women had a higher prevalence of arthritis than men. The prevalence of arthritis was high in subjects with lower education and economic status. Vitamin B1 and iron, potassium and calcium intake in patients with arthritis were lower than those recommended. In particular, pain in patients with arthritis was significantly different according to the recommended intake of vitamin B1 and calcium. Nutrient intake and pain were associated with arthritis. It has been confirmed that nutritional guideline intervention for proper nutrient intake is necessary as an active countermeasure to prevent pain and aggravation of arthritis patients.
\end{abstract}

Keywords: Arthritis patients, Pain, Nutrient intake, Nutrition, Survey

\section{Introduction}

Today, changes in nutritional status and living environment due to the improvement of national income are leading to a rapid increase in chronic diseases. Chronic illness is emerging as a major health problem affecting the competitiveness of individuals as well as families and the nation. In the National Health and Nutrition Survey conducted in Korea, 99.1 patients were affected by arthritis. And the third highest disease prevalence in terms of chronic diseases. Arthritis causes severe pain and causes physical discomfort due to joint deformation. This reduces the social function and vitality of people suffering from arthritic diseases and impairs their quality of life by limiting independent daily living. Patients experiencing arthritis complain that their greatest difficulty is pain. Therefore, the goal of treatment of osteoarthritis is to improve the quality of life by reducing pain and minimizing the daily life impairment of patients. The causes of pain associated with arthritis have not been clearly elucidated. It is known that the pain of muscle or fascia origin, which is assumed to be the cause of arthritis pain, is caused by psychological factors, physical stimulation, metabolic abnormalities, chronic infection, and inadequate nutritional status causing energy supply disturbances. Gerwin suggested that inadequate nutrition and some invisible nutrient deficiencies can cause and exacerbate muscle and fascia pain. Vitamin B1, B6, B12, folic acid, and Vitamin C, which are involved in the energy production of human essential nutrients, and

Article history:

Received (July 21, 2016), Review Result (September 07, 2016), Accepted (October 28, 2016) 
deficiencies such as calcium, iron, and potassium that are essential for normal muscle function are the main causes of pain activation or aggravation. Schwartz and Adamy reported that ingestion of nutrients such as calcium and vitamins and some foods were effective in preventing and alleviating arthritis. Managing nutrient intake in patients with arthritis is likely to affect disease prevention and pain management [1][2][3][4][5][6][7][8][9][10]. However, previous studies on this field are rare. Although there is a high incidence of arthritis in Korea, there have been few studies on the causes of pain and treatment effects in dietary or nutrient intake status. Therefore, it is necessary to analyze the pain and nutrient intake status of

patients with arthritis using the results of the nation's representative representation. Therefore, this study was conducted to investigate the nutritional intake status of arthritis patients according to the fifth National Health and Nutrition Examination Survey data. I want to provide basic data.

\section{Research methods}

\subsection{Study design}

This study is a secondary analysis study using the National Health and Nutrition Examination Survey data from the Disease Control Headquarters.

\subsection{Study subjects}

In this study, we selected 816 subjects who were diagnosed with arthritis as the first subjects who participated in the 5th National Health and Nutrition Examination and responded to Vitamin B1, calcium, iron, and potassium intake of the nutrition survey. Among them, the 403 respondents who answered the pain experience question per year were used for the final analysis.

\subsection{Research tools}

\subsubsection{Sociodemographic characteristics}

Population and sociological variables included gender, age, marital status, education level, monthly income, occupation, subjective health status, osteoarthritis treatment, drinking, smoking. Subjective health status is 'How do you think your health is like your usual?' In response to the question, it was classified as 'very good / good', 'normal', and 'bad / very bad'. The current status of treatment for arthritis is classified as 'not being cured', 'not being cured but not treated', or 'currently being treated' for the item 'Are you currently undergoing treatment?' Drinking is divided into 'Yes / No' in response to the question 'Have you ever drank more than one drink while living so far?' Smoking was categorized as 'Yes / No' in response to the question 'Have you ever smoked one cigarette?' The limit of activity is classified as 'Yes / No' in response to the question 'Are you currently being restricted by your health or physical or mental disability in your daily life and social activities?' Pain was categorized as 'Yes / No' in response to 'Have you experienced symptoms such as pain, stiffness, or swelling around the joints during the past year?'

\subsubsection{Nutrient intake status}


Nutrient intakes were measured by individual daily intake of food intake data using the 24 - hour method of the National Health and Nutrition Survey, and vitamin B1, calcium, iron, and potassium were used for the analysis.

\section{Data collection and data analysis method}

\subsection{Collecting data}

In this study, the 5th National Health and Nutrition Survey was used. The survey period was from January to December 2012. The National Health and Nutrition Survey has secured the reliability of data gathered by researchers and researchers who have been directly trained in the knowledge and considerations required by the researchers who prepared the questionnaire. Based on the problems found in the 2001 National Health and Nutrition Examination Survey, we collected opinions from various experts and revised and supplemented them in each field. After establishing a working committee and consulting expert advisors, the research items were selected by considering the international comparison, and after the preliminary investigation, the final survey items were approved by the National Statistical Office and the reliability and validity of the survey items respectively.

\subsection{Data analysis method}

Data analysis was done using SPSS / win 22.0 program. The concrete method is as follows.

-The chi-square test was used to analyze the differences in the demographic characteristics and pain of the subjects.

-The chi-square test was used to compare the nutritional status of the subjects with the pain.

-The relationship between pain and nutrient intake was analyzed by Pearson correlation.

\section{Results}

\subsection{Population and social characteristics due to pain}

There were statistically significant differences between the patients with painful arthritis and the patients without pain, including gender, age, marital status, education level, monthly income, subjective health status, current osteoarthritis treatment, and activity limitation. In contrast to $22 \%$ of males and $78 \%$ of females with painful arthritis, $36 \%$ of males and $64 \%$ of females had arthritis without pain and $64 \%$ of females had painful arthritis $\left(\chi^{2}=4.686, p=.030\right)$. Age was found in $47 \%$ of patients with painful arthritis aged 65 years or older, and $28 \%$ of painful arthritis patients aged 65 and over were older than 65 years of age with painful arthritis $\left(\chi^{2}=6.402, \mathrm{p}=.011\right)$. Marital status was found in $66 \%$ of patients with painful arthritis and in $83 \%$ of patients with arthritis without pain, with more painful arthritic patients $\left(\chi^{2}=5.492, \mathrm{p}=.019\right)$. The education level of the patients with painful arthritis was $37 \%$ for elementary school graduates and $31 \%$ for the uneducated students, while the patients with painless arthritis were $32 \%$ for elementary school graduates and $30 \%$ for high school graduates $\left(\chi^{2}=23.541, \mathrm{p}=.000\right)$. Monthly income was $88 \%$ for patients with painful arthritis (less than 2 million won), compared with $65 \%$ for arthritis patients without pain (less than 2 million won) $\left(\chi^{2}=7.828, \mathrm{p}=.050\right) .57 \%$ of patients with painful arthritis reported a poor subjective health condition, and $36 \%$ of patients with painless arthritis reported bad $\left(\chi^{2}=12.985, \mathrm{p}=.002\right)$. Currently, only $53 \%$ of patients with arthritis who are currently treated with pain have arthritis, whereas only $13 \%$ of patients with painless arthritis are currently receiving treatment. The current treatment is painful arthritis Many $\left(\chi^{2}=122.816, p=.000\right)$. 
Activity limitations were limited in $27 \%$ of painful arthritis patients, and in pain-free arthritis patients, $6 \%$ of them had activity limitations, which resulted in limited activity in patients with painful arthritis $\left(\chi^{2}=9.884, \mathrm{p}=.002\right)$.

\subsection{Nutrient intake status in patients with painful arthritis and painless arthritis}

Vitamin B1 and Calcium were the statistically significant differences between the patients with painful arthritis and those without pain. Vitamin B1 is the recommended dose of $28 \%$ of patients with painful arthritis, whereas $51 \%$ of patients with painless arthritis recommend a sufficient dose of Vitamin B1 for pain-free arthritis patients $\left(\chi^{2}=10.287, p=.001\right)$. Calcium was the recommended dose of $16 \%$ of patients with painful arthritis, whereas $32 \%$ of patients with painless arthritis consumed Calcium more than the recommended dose of arthritis without pain $\left(\chi^{2}=7.156, \mathrm{p}=.007\right)$.

\subsection{Correlation between pain and nutrient intake status}

Pain was positively correlated with Vitamin B1 ( $\mathrm{r}=.160)$ and Calcium (r=.133).

\section{Conclusion}

Recently, there has been a growing interest in managing and preventing chronic diseases through appropriate nutrient intake. Therefore, there is a need to confirm the relationship between major chronic diseases such as arthritis and nutrient intake in Korea. The National Health and Nutrition Survey data, which systematically investigate individual health, diet and related factors by selecting representative groups of Korean people, may be useful for developing guidelines for health promotion of patients with arthritis. The purpose of this study was to compare the nutritional status, pain, and quality of life of arthritis patients. The prevalence of arthritis in women is $77 \%$, which is higher than that of men. This is consistent with the previous studies that arthritis prevalence is higher in women than in men [11][12]. In addition, the lower the economic level and the lower the level of education, the higher the prevalence of arthritis, which supports the existing research results [13]. These results suggest that it is necessary to develop an arthritis management program for women with low economic and educational levels. According to nutrient intakes, $63.5 \%$ of patients with arthritis consumed less than the recommended amount of vitamin B1, $44.3 \%$ of iron, $32.4 \%$ of potassium, and $85 \%$ of calcium were consuming less than the recommended amount. In the study of Kim et al., which reported that $63.5 \%$ of patients with arthritis had Vitamin B1 deficiency in calcium, vitamin B1 and iron in the study of elderly arthritis patients, And Chang et al. suggest that daily intake of calcium, Vita-min B1, iron, and potassium in arthritis subjects is lower than that of Korean adults. Therefore, a plan to improve the nutrient intake of patients with arthritis should be established. As a result of comparing the presence of pain and each nutrient intake, the pain of arthritis patients was significantly different according to the recommended amount of vitamin B1 and calcium intake. In other words, Vita-min B1 and calcium were consumed less than the recommended amount than the group without pain. Arthritis is reported to cause pain due to muscle damage or fascia damage [14]. The results of this study are consistent with the finding that inadequate nutrition, deficiency of vitamins and minerals cause and exacerbate pain in patients with muscle pain or fascia pain [15]. On the other hand, the pain of arthritis patients did not show any significant difference according to the recommended intake of potassium and iron. These results are in contrast to the findings of Gerwin RD and Gerwin RD \& Gevirtz R. that iron and potassium, which produce and supply intramuscular energy, are associated with pain induction and exacerbation. Research into the 
relationship between pain and certain nutrients such as vitamins and minerals in arthritis patients has not been available in Korea until now. Therefore, the follow-up study should be continued to find out the relationship between pain and nutrition in patients with arthritis. It is necessary to develop and apply nutritional guidelines to prevent and control the pain and aggravation of arthritis patients.

\section{References}

[1] Lim, N. Y. and Lee E. Y, "Functional impairment and psychological status in patients with chronic arthritis," The Journal of Rheumatology Health, vol.5, no.1, pp.72-82, (1998)

[2] Blixen, C, Kippes, and C, "Depression, social support, and quality of life in older adults with osteoarthritis," Image Journal of Nursing Scholarship, vol.31, no.3, pp.221-226, (1998) DOI:10.1111/j.15475069.1999.tb00484.x

[3] J. Hallberg, "Pain and quality of life among older people with rheumatoid arthritis and/or osteoarthritis," Journal of Clinical Nursing, vol.11, no.4, pp.430-443, (2002)

[4] Lee and H. B, "The behavior of complementary and alternative medicine used by hospitalized patients with degenerative arthritis," M.S. thesis, Kyungpook University, (2007)

[5] Rejeski, W. J., Focht, B. C., Messier, S. P., Morgan, T., Pahor, M., Pennin, and B. "Obese, older adults with knee osteoarthritis," Weight loss, exercise and quality life, Health Psychology, vol.21, no.5, pp.419-426, (2002)

[6] Gerwin and R. D., "A study of 96 subjects examination both for fibromyalgia and myofascial pain," Journal of musculoskeletal pain, vol.3, no.121, (1995)

[7] Joosten, E., Van den Berg, and A., "Metabolic evidence that deficiencies of vitamin B-12(cobalamin), folate, and vitamin B-6 occur commonly in elderly people," American Journal of Clinical Nutrition, vol.58, pp.468476, (1993) DOI:10.1093/ajcn/58.4.468

[8] Schwartz ER and Adamy L., "Effect of ascorbic acid on arylsulfatase activities and sulfated proteoglycan metabolism in chondrocyte cultures," Journal of Clinical Investigation, vol.60, pp.96-106, (1997) DOI:10.1172/JCI108774

[9] Kim and H. A., "Nutritional management and weight control of patient with osteoarthritis," The Journal of Rheumatology Health, vol.2, no.2, pp.218-226, (1995)

[10] Chang and O. J., "A study on nutrient intake status and serum minerals in Korean osteoarthritis women," Ph.D. dissertation, Soon chunghyang University, Seoul, (2005)

[11] Yang, H. J. and Park, J. S., "The study of pain, depression and quality of life according to the coping strategy in chronic arthritis patients," The Journal of Korean Academic Society of Adult Nursing, vol.14, no.2, pp.213-221, (2002)

[12] Yoo and M. C., "New perspectives of treatment of osteoarthritis," The Journal of Rheumatology Health, vol.2, no.2, pp.227-229, (1995)

[13] Chung, S. K., Nam, C. H., Kim, S. S., Jeon, B. C., Kim, G. Y., Ha, E. P., Moon, and K. N., “Arthritis patient' knowledge level and it's related factors," Journal of Korean Society for Health Education and Promotion, vol.19, no.1, pp.31-43, (2002)

[14] Delisa, J. A., Gans, and B. M., "Rehabilitation medicine-principles and practice," (3rd Edition), Philadelphia, New York: Lippincott-Raven, (1998) DOI:10.1016/0363-5023(94)90291-7

[15] Gerwin, R. D., Gevirtz, and R., "Chronic myofascial pain: iron insufficiency and coldness as risk factors," Journal of musculoskeletal pain, vol.3, no.120, (1995) 
Nutrient Intakes According to Pain in Patients with Arthritis

This page is empty by intention. 